\title{
Partially Hydrolyzed Whey Formula Intolerance in Cow's Milk Allergic Patients
}

\author{
Maureen Egan, MD ${ }^{1}$, Tricia Lee, MD² $^{2}$, Jade Andrade, BS $^{2}$, Galina Grishina, $\mathbf{M S}^{2}$, Michelle \\ Mishoe, BA ${ }^{2}$, Gustavo Gimenez, MS $^{2}$, Hugh A Sampson, MD $^{2}$, and Supinda Bunyavanich, \\ MD, MPH ${ }^{2,3,{ }^{*}}$ \\ ${ }^{1}$ Section of Pediatric Allergy and Immunology, Department of Pediatrics; Children's Hospital \\ Colorado, Aurora, CO, USA \\ 2Division of Allergy and Immunology, Department of Pediatrics; The Icahn School of Medicine at \\ Mount Sinai, New York, NY, USA \\ ${ }^{3}$ Department of Genetics and Genomic Sciences and Mindich Child Health and Development \\ Institute, Icahn School of Medicine at Mount Sinai, New York, NY, USA
}

\section{Keywords}

Food Allergy; Cow's Milk Allergy; Hypoallergenic Formula; Partially Hydrolyzed Whey Formula

\section{To the Editor}

Cow's milk allergy (CMA) is one of the most common food allergies in young children, affecting $2-3 \%$ of the pediatric population (1). Hypoallergenic formula is recommended for infants with CMA who are not breastfeeding (2). Extensively hydrolyzed or amino acidbased formulas are tolerated in over $90 \%$ of infants with CMA(2). However, these formulas cost more than standard formulas and are less palatable (2).

CMA is due to immunoglobulin E (IgE) production against proteins in cow's milk (3). The major proteins in cow's milk are casein (alphaS1-, alphaS2-, beta-, and kappa-caseins) and whey (alpha-lactalbumin, beta-lactoglobulin, bovine lactoferrin, bovine serum albumin, and bovine immunoglobulins) (3). Caseins, alpha-lactalbumin (ALA), and beta-lactoglobulin (BLG) are the major cow's milk allergens, with many individuals co-sensitized (3).

Partially hydrolyzed whey formula (pHWF) is not currently recommended for patients with CMA(2). Prior European studies evaluating the allergenicity of infant formulas reported that

\footnotetext{
*Address correspondence to Supinda Bunyavanich, MD, MPH, 1425 Madison Avenue \#1498, New York, NY 10029, tel. (212) 659-8262, fax (212) 426-1902, supinda@ post.harvard.edu.

Maureen Egan, MD, Children's Hospital of Colorado, Aurora, CO, USA

Tricia Lee, MD, Icahn School of Medicine at Mount Sinai, New York, NY

Jade Andrade, Icahn School of Medicine at Mount Sinai, New York, NY

Galina Grishina, Icahn School of Medicine at Mount Sinai, New York, NY

Michelle Mishoe, Icahn School of Medicine at Mount Sinai, New York, NY

Gustavo Gimenez, Icahn School of Medicine at Mount Sinai, New York, NY

Hugh A Sampson, MD, Icahn School of Medicine at Mount Sinai, New York, NY

Supinda Bunyavanich, MD, MPH, Icahn School of Medicine at Mount Sinai, New York, NY
} 
40-60\% of patients with CMA can tolerate pHWF (4-6). However, these studies were performed on sample sizes of 8 to 25 subjects over 15 years ago (4-6), and current rates may differ. In this study, we sought to characterize the current prevalence of pHWF tolerance among US patients with CMA.

We previously described the immunologic profile of a child with CMA who tolerated pHWF(7). This patient was sensitized to casein only, which was consistent with her tolerance of whey-based pHWF. This case suggested that there could be clinical utility to assessing sensitization to individual cow's milk proteins because doing so could identify CMA individuals able to take pHWF (2,7). Prior studies evaluating tolerance to pHWF in CMA patients did not examine cow's milk component testing in relation to pHWF tolerance (4-6). An additional goal of this study was to identify the clinical and immunologic characteristics of patients with CMA who tolerate pHWF.

\section{Methods}

Participants

All patients age 6 months to 18 years with CMA seen at the pediatric allergy/immunology practices at the Mount Sinai Health System (New York, NY, USA) between March 2015December 2015 were eligible and invited to participate. CMA was defined as an allergic reaction to milk within 6 months before study entry, and a milk-specific $\operatorname{IgE}(\operatorname{sgE})$ level or skin prick test (SPT) highly predictive for clinical reactivity $\left(\mathrm{sIgE}>2 \mathrm{kU} \mathrm{A}_{\mathrm{A}} / \mathrm{L}\right.$ if $\leq$ age 2 years, $\operatorname{sIgE}>5 \mathrm{kU}_{\mathrm{A}} / \mathrm{L}$ if $>$ age 2 years, or SPT wheal diameter $\geq 3 \mathrm{~mm}$ ).

\section{Oral food challenges}

All subjects underwent an OFC to pHWF (Gerber Good Start ${ }^{\circledR}$ ) with administration at 15 minute intervals of incremental amounts $(15 \mathrm{ml}, 30 \mathrm{ml}, 75 \mathrm{ml}, 120 \mathrm{ml})$ for a total of $240 \mathrm{ml}$, a typical serving of cow's milk. Gerber Good Start $®$ is marketed as a $100 \%$ whey proteinbased formula, although it is not marketed or recommended for children with CMA (2). In children in whom anxiety may have contributed to symptoms, single-blinded placebo doses were also administered. If major signs or symptoms of an allergic reaction arose, the challenge was stopped and treatment was administered.

\section{Immunoblotting}

Immunoblotting against cow's milk and pHWF was performed with serum from each patient and a non-atopic individual as a negative control (NC). Alkaline phosphatase-conjugated goat-anti human IgE was used as a secondary antibody. Immunoblots were developed with phosphatase substrate.

\section{SPT and sigE testing to milk and milk components}

All subjects underwent SPT to 1:1 dilution of pHWF; 1:10 dilution of pHWF; casein, BLG, cow's milk, saline and histamine. Serum testing was also performed via Phadia ImmunoCAP included specific IgE (sIgE) to cow's milk, BLG, ALA and casein. These were done prior to the OFC by a blinded physician. 


\section{Epitope studies}

A Luminex-Based Peptide Assay (LPA) was performed using serum from each participant, an atopic control without CMA, and two pools of sera from patients with CMA. We examined binding to cow's milk epitopes that were previously found to be informative in CMA, including epitopes from casein (as1-casein, as2-casein, b-casein and k-casein) and whey proteins (b-lactoglobulin) (BLG) (9). Analyses for differential epitope binding based on sensitization (using sIgE and SPT to cow's milk, casein, BLG, ALA, and pHWF each as variables) were performed using DESeq2. $\mathrm{P}$ values were adjusted for multiple comparisons using the Benjamini-Hochberg method.

\section{Results}

\section{Oral Food Challenge Results}

The first 10 subjects all had reactions to pHWF during OFC, indicating lack of tolerance. Characterization of the reactions including symptoms and amount ingested are summarized in Table I. There were no serious adverse events.

Statistical analysis showed that with 10 experimental subjects and an observed reaction rate of $100 \%$, additional recruitment was statistically unlikely to yield different outcomes (i.e. pHWF tolerance) at $>99 \%$ power. Given risks to subjects from continued symptomatic OFCs we ended recruitment after these 10 patients all failed to tolerate pHWF.

\section{Clinical characteristics and immunologic profiles of the participating subjects}

The participants ranged from toddlers to school-age children (Table 1) with the majority male (80\%) and Caucasian (80\%). Half had a family history of atopic conditions, and $60 \%$ reported comorbid atopic dermatitis and asthma. None of the patients were tolerating baked milk at the time of study entry, which differs from prior studies reporting tolerance of baked milk in $75 \%$ of patients with CMA (8).

\section{Immunoblots}

Immunoblots of patient serum against cow's milk demonstrated binding to proteins at $14 \mathrm{kDa}, 18 \mathrm{kDa}$ and $220 \mathrm{kDa}$, corresponding to ALA, BLG and casein and its aggregates, respectively (Figure 1A). Immunoblots of patient serum against pHWF did not demonstrate binding to proteins at $14 \mathrm{kDa}$ and $18 \mathrm{kDa}$, corresponding to ALA or BLG respectively, in the majority of patients (Figure 1B). However, $80 \%$ of patients demonstrated binding to molecular proteins $20 \mathrm{kDa}$, corresponding to casein and its aggregates which were shown to be present in pHWF..

\section{Sensitization to milk and milk components}

SPT and SIgE results of the individual patients as well as summary statistics are shown in Table I. Participants 1-7 were co-sensitized to casein and whey proteins, participants 8 and 10 had a predominately casein-specific sensitization, while participant 9 had a predominantly whey-specific sensitization. 
Pearson correlations between skin and serum sIgE test results are shown in Figure 2A. Within our sample, skin and serum test results were not significantly correlated with one another. Significant correlations were only seen within testing modality (sIgE or SPT). sIgE to cow's milk was highly correlated with sIgE to casein and BLG.

\section{Differential epitope binding by Luminex-based peptide assay}

Eleven cow's milk epitopes had differential binding (Padj < 0.05$)$ associated with casein sIgE and/or cow's milk SPT (Figure 2B). Ten of these 11 were casein epitopes.

\section{Discussion}

We observed $0 \%$ tolerance of pHWF among 10 US children with CMA, which is in contrast to previously reported rates of 40-60\% tolerance in European children with CMA (4-6). We ended this study early because of the low tolerance rate and statistical determination that further recruitment was unlikely to yield different outcomes in our patient population. SPT and sIgE profiling to cow's milk and components ranged widely among the study participants. Immunoblots and LPA suggested that residual caseins in pHWF were responsible for our patients' allergic reactions to pHWF.

The small sample sizes of our and previous studies (4-6) of pHWF in CMA patients, as well as population differences (e.g. European vs. US, temporal differences in milk allergy) may account for the disparate rates observed. Additionally, given our strict inclusion criteria and referral population, it is possible that our study population had more severe CMA phenotypes.

In contrast to previously published studies examining pHWF tolerance in CMA individuals (4-6), we rigorously characterized participants' milk component and immunologic profiles via immunoblot and LPA. Rugo et al. reported positive SPT to pHWF in $6(35 \%)$ of 17 individuals, detectable sIgE to pHWF in 7 (70\%) of 10 individuals, and clinical reactions to pHWF on OFC in 5 (64\%) of 8 participants (6). Giampietro et al. reported $10(32 \%)$ of 31 subjects with CMA had a positive SPT to pHWF with $9(36 \%)$ of 25 participants reacting to pHWF on OFC (4). Ragno et al. demonstrated positive SPT to pHWF in $9(45 \%)$ of 20 subjects, positive sIgE to pHWF in 13 (65\%) of 20 subjects, and 9 (45\%) of 20 children reacting to $\mathrm{pHWF}$ on OFC (5). Because most of these previous studies only reported sensitization to whole cow's milk and pHWF with no component or other analyses, conclusions regarding the immunologic phenotype of CMA patients tolerant of pHWF could not be made (4-6).

The milk component testing we performed included SPT and sIgE to casein and whey proteins (BLG and ALA), the main proteins in cow's milk (3). Based on this component testing, we were able to identify patients with casein-predominant CMA, whey-predominant CMA, and casein and whey co-sensitization. Despite this range of sensitization phenotypes, no participant tolerated pHWF. Our results suggest that examination of milk components is not helpful in identifying CMA patients tolerant of pHWF. Our initial hypothesis, based on a prior case report (7) of a child with casein-specific allergy and tolerance of pHWF was that individuals with casein-specific SPT and SIgE would pass their pHWF OFC. This was not 
the case, as participant numbers 8 and 10 fit this optimal sensitization profile and did not tolerate pHWF.

The immune profiling we conducted (immunoblots and LPA) provided additional information that previous studies lacked. Immunoblotting of patient serum against pHWF (Figure 1) demonstrated binding to casein and its aggregates, which one would expect to be essentially absent in pHWF. The presence of residual casein in pHWF has been reported previously $(6,10)$. Sampson et al. performed studies comparing pHWF (Gerber Good Start $\left.{ }^{\circledR}\right)$ to casein hydrolysate (Alimentum ${ }^{\circledR}$ ) and found that pHWF had 100 times the amount of detectable casein and 700 times the amount of detectable whey proteins compared to Alimentum ${ }^{\circledR}$ (10). Specifically, pHWF was noted to have identifiable casein protein in the range of $15.5-38.2 \mu \mathrm{g} / \mathrm{ml}$ compared to $0.63-0.71 \mu \mathrm{g} / \mathrm{ml}$ in Alimentum (10). Additionally, SDS analysis of pHWF clearly demonstrated peptide fractions $>4 \mathrm{kDa}$, with it being known that peptides $>3.4 \mathrm{kDa}$ can produce allergic symptoms (10). More recently, Lee et al. reported binding to casein proteins on immunoblots of pooled sera from subjects with CMA against pHWF (7). Similarly, Catala-Clariana et al. identified casein proteins, specifically Bcasein, as1-casein, as2-casein and k-casein, to be present in pHWF as well as whey protein, BLG (11). We hypothesize that residual casein contributed to the lack of tolerance to pHWF observed in our study, particularly in the patients with a predominantly casein-specific CMA.

By including LPA in our immune profiling of subjects, we demonstrated statistically significant differential binding of 10 casein protein epitopes based on cow's milk SPT and casein SIgE. Only one whey protein epitope, betalac- 15 , was found to have a statistically significant association with cow's milk SPT. The differential binding of primarily casein epitopes highlights the predominance of casein sensitization and further supports the concept of residual casein contributing to lack of pHWF tolerance.

This study has several strengths. Reactivity to pHWF was confirmed for all participants based on OFC. Thorough immunologic evaluations, specifically cow's milk components, were performed to characterize participants' CMA. Our study population comprised a range of clinical CMA with all relevant phenotypes (casein and whey co-sensitization, casein predominant and whey predominant sensitization) included. Immunoblots and LPA further clarified the lack of tolerance to pHWF in patients with casein-predominant sensitization by demonstrating the presence of residual casein in pHWF.

We recognize this study's limitations. First, while our study sample was small, statistical calculations based on all 10 subjects reacting to pHWF indicated that there was no marginal benefit to continued recruitment. The component correlation and epitope analyses may have yielded additional findings with a larger sample. Second, there was likely referral bias in our participants, as the subjects' uniform lack of tolerance to baked milk at study entry suggests a bias towards a more severe CMA phenotype that may be less amenable to any degree of tolerance. A study of pHWF tolerance in baked milk-tolerant CMA children is worthwhile to consider. Our cohort spanned a range of ages, and our findings may have differed had our population included only young children. Finally, because our single-center cohort was 
predominantly male and Caucasian, additional studies are needed to assess the generalizability of our findings to other populations.

\section{Conclusions}

Our findings support current recommendations that partially hydrolyzed whey formula should not be recommended for individuals with CMA (2). While there are reported individuals with CMA tolerant of pHWF (4-7), our current US-based study suggests that the prevalence of $\mathrm{pHWF}$ tolerance is far lower than previously reported in earlier European studies. Our study also indicates that, at present, there is no reliable immunologic testing to identify these individuals. Our findings additionally suggest that evaluation of $\mathrm{CM}$ components is not clinically beneficial in the routine evaluation of CMA.

\section{Acknowledgments}

Funding: Nestle provided partial support for this investigator-initiated study, including Gerber Good Start formula and funding for skin test reagents and sIgE assays. They had no role in the design, implementation, analysis, or writing of results. SB is supported by the National Institutes of Health R01 AI118833.

The authors would like to thank Ronaldo Silva for statistical assistance with the manuscript.

\section{Abbreviations}

$\begin{array}{ll}\text { ALA } & \text { Alpha-lactalbumin } \\ \text { AP } & \text { Alkaline Phosphatase } \\ \text { BLG } & \text { Beta-Lactoglobulin } \\ \text { CMA } & \text { Cow's Milk Allergy } \\ \text { IgE } & \text { Immunoglobulin E } \\ \text { LPA } & \text { Luminex-based Peptide Assay } \\ \text { NC } & \text { Negative Control } \\ \text { OFC } & \text { Oral Food Challenge } \\ \text { pHWF } & \text { Partially Hydrolyzed Whey Formula } \\ \text { SPT } & \text { Skin Prick Testing } \\ \text { SIgE } & \text { Specific IgE }\end{array}$

\section{References}

1. Skripak JM, Matsui EC, Mudd K, Wood RA. The natural history of IgE-mediated cow's milk allergy. J Allergy Clin Immunol. 2007 Nov; 120(5):1172-1177. [PubMed: 17935766]

2. American Academy of Pediatrics. Committee on Nutrition. Hypoallergenic infant formulas. Pediatrics. 2000 Aug; 106(2 Pt 1):346-349. [PubMed: 10920165]

3. Wal JM. Bovine milk allergenicity. Ann Allergy Asthma Immunol. 2004 Nov; 93(5 Suppl 3):S2-11. [PubMed: 15562868] 
4. Giampietro PG, Kjellman NI, Oldaeus G, Wouters-Wesseling W, Businco L. Hypoallergenicity of an extensively hydrolyzed whey formula. Pediatr Allergy Immunol. $2001 \mathrm{Apr}$; 12(2):83-86. [PubMed: 11338291]

5. Ragno V, Giampietro PG, Bruno G, Businco L. Allergenicity of milk protein hydrolysate formulae in children with cow's milk allergy. Eur J Pediatr. 1993 Sep; 152(9):760-762. [PubMed: 8223811]

6. Rugo E, Wahl R, Wahn U. How allergenic are hypoallergenic infant formulae? Clin Exp Allergy. 1992 Jun; 22(6):635-639. [PubMed: 1393761]

7. Lee TD, Gimenez G, Grishina G, Mishoe M, Sampson HA, Bunyavanich S. Profile of a milkallergic patient who tolerated partially hydrolyzed whey formula. J Allergy Clin Immunol Pract. 2015 Jan-Feb;3(1):116-118. [PubMed: 25577632]

8. Nowak-Wegrzyn A, Bloom KA, Sicherer SH, Shreffler WG, Noone S, Wanich N, et al. Tolerance to extensively heated milk in children with cow's milk allergy. J Allergy Clin Immunol. 2008 Aug; 122(2):342-7. 347.e1-2. [PubMed: 18620743]

9. Sackesen C, Lin J, Schmidt S, Getts RC, Kadushin J, Gimenez G, et al. A New Luminex-Based Peptide Assay to Identify Different Degrees of Milk Allergic Reactivity. J Allergy Clin Immunol. 2015; 2(135):AB36.

10. Sampson HA, Bernhisel-Broadbent J, Yang E, Scanlon SM. Safety of casein hydrolysate formula in children with cow milk allergy. J Pediatr. 1991 Apr; 118(4 Pt 1):520-525. [PubMed: 2007924]

11. Catala-Clariana S, Benavente F, Gimenez E, Barbosa J, Sanz-Nebot V. Identification of bioactive peptides in hypoallergenic infant milk formulas by CE-TOF-MS assisted by semiempirical model of electromigration behavior. Electrophoresis. 2013 Jul; 34(13):1886-1894. [PubMed: 23564639] 


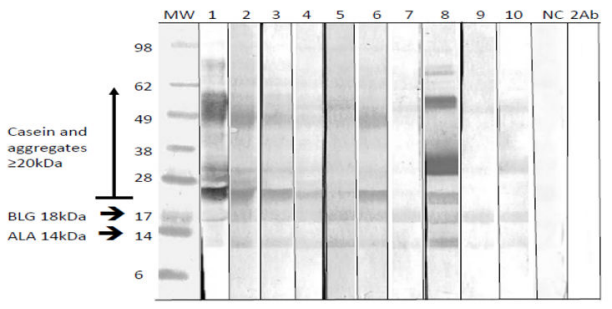

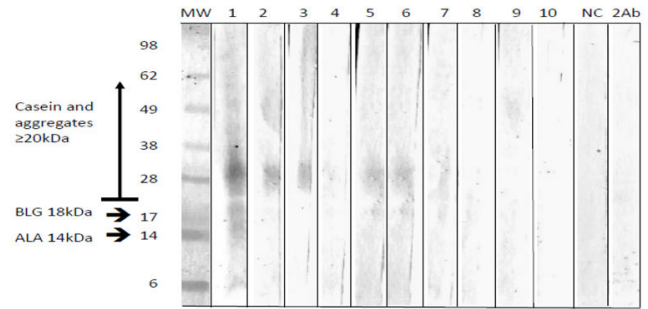

Figure 1. Immunoblots of patients' serum against cow's milk (A) and partially hydrolyzed whey formula $(B)$

A. All subjects demonstrated binding to casein and whey proteins (BLG and ALA) present in cow's milk. The negative control (NC) and secondary antibody control (2Ab) did not show any binding. B. Subjects demonstrated binding to casein, supporting residual casein in pHWF. No subjects demonstrated binding to whey proteins. The negative control (NC) and secondary antibody control $(2 \mathrm{Ab})$ did not show any binding. $\mathrm{MW}=$ molecular weight ladder (kDa). 


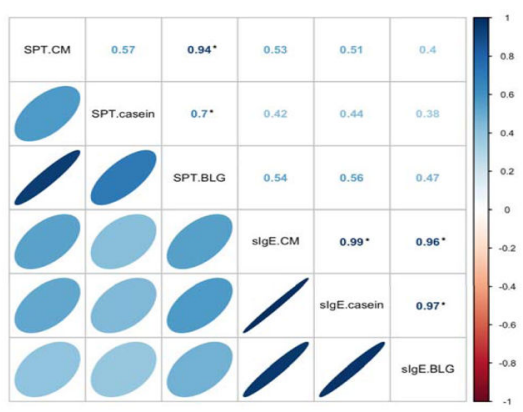

B

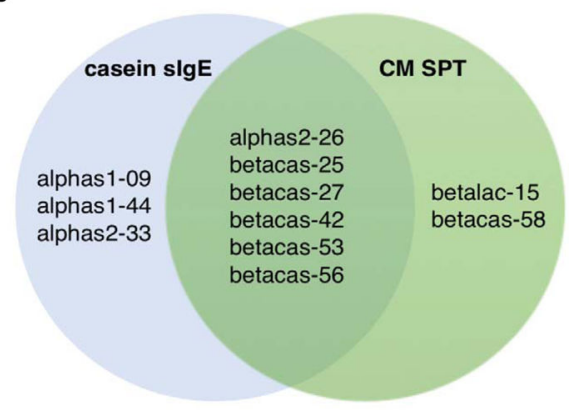

Figure 2. Component and LPA epitope analyses of CMA patients

A: Correlation matrix between skin prick testing (SPT) and serum specific IgE levels to cow's milk and components. Labels are shown along the diagonal. Pearson correlation $\mathrm{r}^{2}$ values are shown in the upper right, with significant correlations $(\mathrm{P}<0.05)$ marked with *. The color of these values, as well as the color and eccentricity of the ellipses in the bottom left, correspond to $\mathrm{r}^{2}$ values as shown by the vertical legend on the right. B: Milk epitopes significantly associated with casein specific $\operatorname{IgE}$ (sIgE) and/or cow's milk skin prick test (CM SPT) at $\mathrm{P}_{\mathrm{adj}}<0.05$ are shown in this Venn diagram. 
